EARTH ROTATION IINFORMATION DERIVED FROM MERIT

AND POLARIS VLBI OBSERVATIONS

D. S. Robertson and W. E. Carter

National Geodetic Survey, National Ocean Survey, NOAA

Rockville, Maryland 20852 U.S.A.

ABSTRACT. In September and October 1980, the National Geodetic Survey, jointly with the National Aeronautics and Space Administration and several other agencies and institutions, conducted a series of astronomical radio interferometry (VLBI) observing sessions to support the IAU/IUGG MERIT short campaign. A total of $14^{\circ}$ days of observations, organized into two 7-day sessions, was collected by three observatories in the United States (Harvard Radio Astronomy Station (HRAS), Haystack Observatory, and Owens Valley Radio Observatory) and the Onsala Space Observatory in Sweden. Chilbolton Observatory, England, and Effelsberg Observatory, West Germany, also participated on some days. Immediately following the MERIT canpaign, NGS initiated a series of 24-hour observing sessions, spaced at

approximately 2-week intervals, as a pilot program to project POLARIS. All of these sessions included two observatories, HRAS and Haystack, and Onsala participated in about half of the sessions. The MERIT and POLARIS

observations were made with the third generation MARK III VLBI system using procedures and schedules designed to yield high quality geodetic

information, including Earth rotation values. This paper briefly traces the planning, observing, and data processing activities, and presents the Earth rotation information thus far derived from the data.

\title{
INTRODUCTION
}

The National Geodetic Survey (NGS) is striving to improve the temporal and spatial resolutions of geodetic measurements to better serve modern Earth sciences. Our immediate goal is to develop the ability to monitor geometrical variations in survey networks of regional, continental, and global scale with a spatial resolution of a few centimeters and a temporal resolution of better than one day. This goal can only be achieved by introducing improved methods of measurement which exploit technological advances stimulated by the space exploration program. Since these advanced methods generally involve observations of extraterrestrial objects such as artificial satellites or quasars, it is necessary to account for variations in the orientation of the Earth in space in reducing and interpreting the observations. 
Traditionally the orientation of the Earth has been determined from optical stellar observations which relate the spin axis and the origin of longitude to a reference frane defined by a specific star catalog. Several difficulties, including the limited observational accuracy imposed by atmospheric refraction and the complexities of accounting for the proper motions of the stars, argue against atteupting to improve the classical system to the accuracy required for modern geodesy. Fundamentally more powerful observational techniques and a more nearly inertial reference frame are required. Independent clock radio interferometry (commonly referred to as Very Long Baseline Interferometry or VLBI) observations of extragalactic radio sources addresses both requi rements simultaneously. This line of reasoning led NGS in 1977 to initiate project POLARIS (Polar-motion Analysis by Radio Interferometric Surveying); (Carter and Strange, 1979; Carter et al., 1979; Carter, 1979). Under project POLARIS, NGS is developing a three-station network of fixed observatories to regularly monitor the orientation of the Earth with respect to a reference frane defined by a selected set, or catalog, of extragalactic radio sources. The first POLARIS observatory, the Harvard Radio Astronomy Station (HRAS), located near Ft. Davis, Texas, reached linited operational status in 1980 .

During September and October 1980 the NGS, jointly with the National Aeronautics and Space Administration (NASA), conducted a series of observing sessions at HRAS, Owens Valley Radio Observatory (OVRO), California, and Haystack Observatory, Massachusetts, to support the International Astronomical Union (IAU)/International Union of Geodesy and Geophysics (IUGG) jointly sponsored MERIT (Monitor Earth Rotation and Intercompare the Techniques of Observation and Analysis) program. European and Massachusetts Institute of Technology researchers organized cooperative efforts at Chilbolton Observatory, England; Effelsberg Observatory, Federal Republic of Germany; and the Onsala Space Observatory, Sweden. The participation of Onsala was particularly important because they were able to schedule all 14 days of the MERIT campaign, and because of their historical and continuing support of geodetic programs.

Immediately following the completion of the MERIT observations, as a pilot program to project POLARIS, NGS initiated a continuing series of 24 hour observing sessions at approximately 2-week intervals using HRAS and Haystack Observatories, supplemented as often as other usage permits by the Onsala Space Observatory. This is the first attempt to use the state-of-the-art MARK III VLBI system operationally. Certain components of the instrumentation and software systems, as well as the operating procedures, are still under development, but many of the fundamental characteristics of radio interferometric surveying are already taking forn. In this paper we trace the flow of activities through a "typical" measuring session, using real data from the MERIT and POLARIS projects for illustrations. The final products, i.e., polar motion $X$ and $Y$ components, UT1, and interstation vectors, are representative of the products which we expect to produce routinely when project POLARIS reaches maturity. 
Establishing and maintaining a project as complex as POLARIS requires extensive planning, fiscal actions, procurements, contracts, and interagency and international coordination. We will not detail these activities here, but emphasize that they are prerequisites to the technical activities and represent a sizeable portion of the total resources invested in the project.

Figure 1 is a simplified functional diagram showing the centers of activities and the primary routes and media of communications for the technical aspects of project POLARIS. The process is highly automated, employing Hewlett Packard HP-1000 computer systems at each of the major centers of activities; i.e., the project operating center, each observatory, and the correlator facility. The communications are predominately in machine-readable forms, including floppy disks, nagnetic tapes, and telecommunications.

The sequence begins at NGS headquarters in Rockville, Maryland. Working within current administrative and technical constraints, the observing instructions and schedules are made and then distributed to the observatories. The observatories nake the observations and forward the data to the correlator facility at Haystack Observatory, Massachusetts. The data tapes are processed through the correlator and the "observables" are sent to the operating center at NGS headquarters. The correlator facility also archives the correlator output and returns the data tapes to the observatories for re-use. When the observational data arrive at NGS they are immediately screened for obvious deficiencies, placed in the NGS VLBI data base, and certified for release to users in NGS and to geodetic specialists in other organizations. Within NGS the data are subjected to detailed analyses and interpretation and the results are reported to the scientific community through oral presentations and technical publications.

\section{OBSERVING STRATEGY}

The VLBI observations of primary importance to geodesy are the delay and delay rate (rate of change of the delay) between the time that a wavefront arrives at the reference point of one radio telescope and the time it arrives at the reference point of the other radio telescope. Because the signals are very weak (usually less than a few Jansky, $10^{-26}$ watts per square meter per Hertz), it is necessary to combine a few tens to a few hundreds of seconds of data to derive each delay and delay rate. A model is used to account for the changes in these values during the observing interval, i.e., to relate the entire span of data to a common epoch, so that each delay and delay rate is actually a "normal point" value. Choosing the duration and content of each observation is the first step necessary in formulating an observing strategy.

The MARK III VLBI system is highly flexible, allowing the user wide latitude in observing strategies. There are 28 channels, each capable of recording at a maxinum rate of 4 megabits per second, that can be operated 


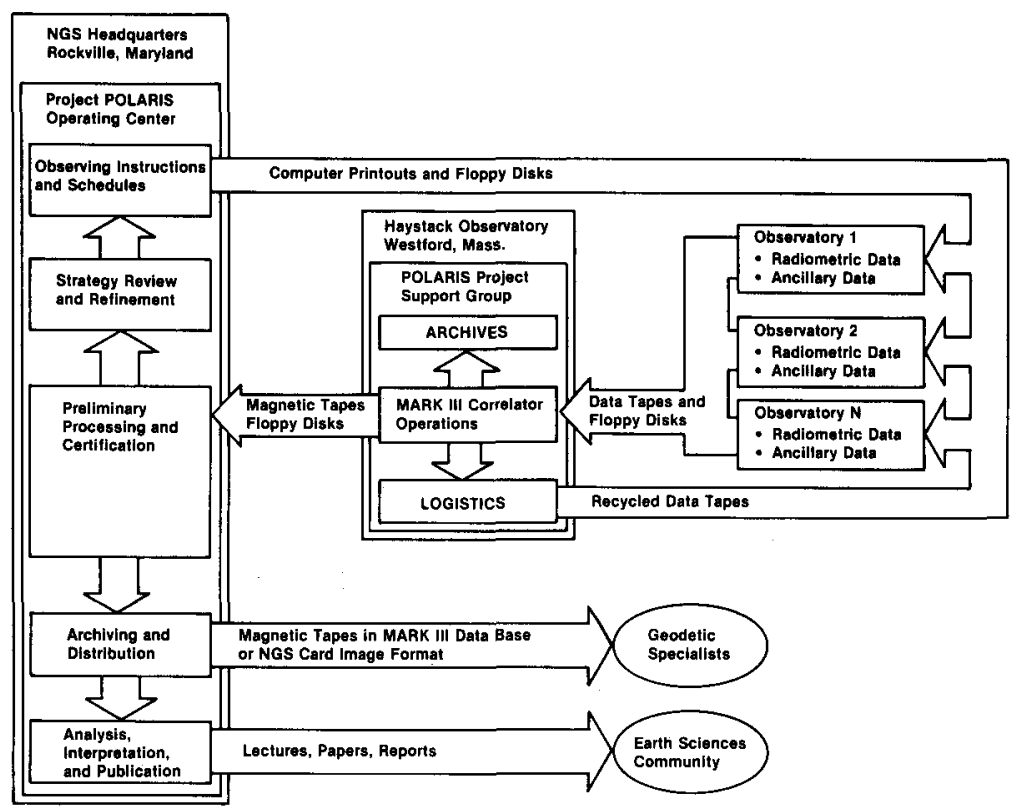

Figure 1. Functional diagram showing centers of activity and primary communication routes and media for project POLARIS.

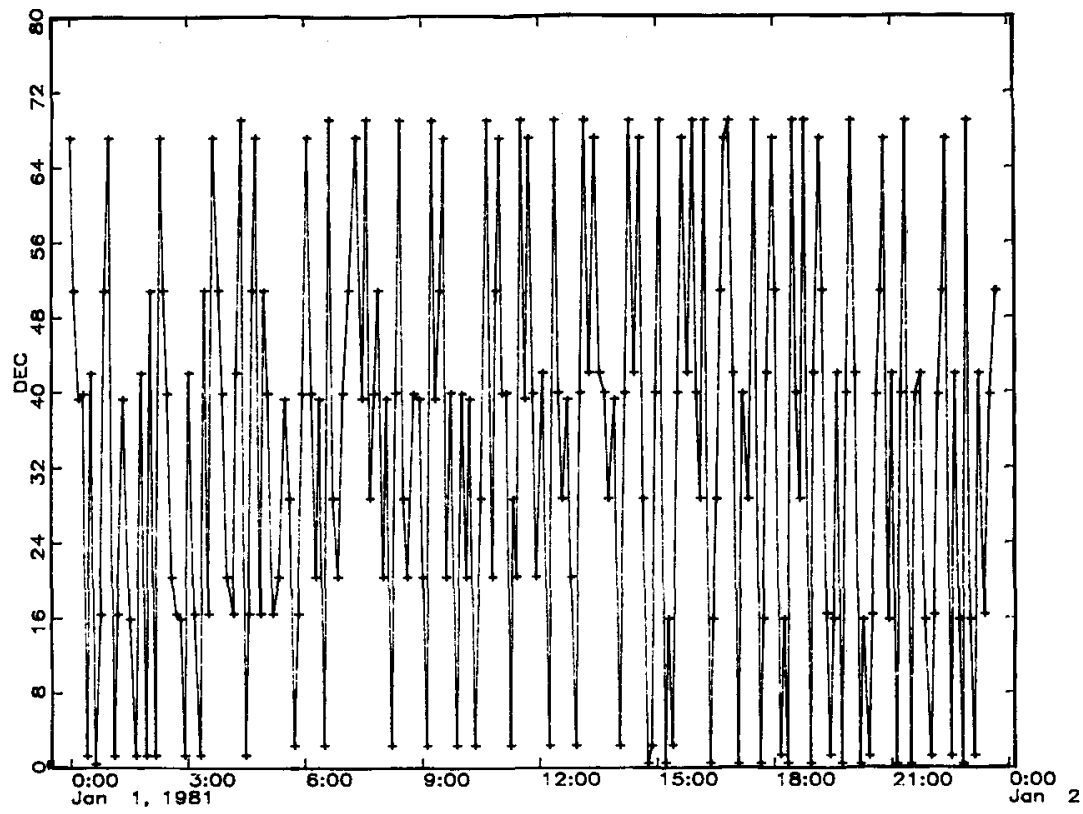

Figure 2. POLARIS observing schedule, showing declinations of observed sources as a function of time. 
in parallel to realize a maximum recording rate of 112 megabits per second. At the maximun recording rate, a 9200 foot long instrumentation tape lasts only about 15 minutes. Since the tapes are costly to purchase and transport, there is an incentive to minimize the amount of tape consumed per observation.

Three factors dictate the minimum quantity of tape required per observation: 1) recording enough bits at each frequency to attain a signal-to-noise ratio (SNR) sufficient to determine the interferometric phase to the desired precision, 2) recording an array of frequencies for bandwidth synthesis, and 3 ) recording $S-$ and $\mathrm{X}$-band data for computing and removing the ionospheric delay.

The relationship between the precision of a given phase measurement and the number of bits, $\mathrm{N}_{f}$, recorded was derived by Rogers (1970) and can be written as follows:

$$
\mathrm{N}_{\mathrm{f}} \simeq\left[\frac{\pi \mathrm{k} T}{\varepsilon \mathrm{A} \sigma_{\phi}}\right]^{2}
$$

Eq. 1

where:

$k$ is Boltzinann's constant,

$\mathrm{T}$ is the geometric mean of the noise temperatures of the two receiving systems,

$\varepsilon$ is the geometric mean aperture efficiencies of the two antennas,

$A$ is the geometric mean collecting area of the two antennas,

$F$ is the correlated flux density received from the radio source at frequency $\mathrm{f}$, and

$\sigma_{\phi}$ is the acceptable standard deviation of the phase measurenent error for a single observation.

In principle, it would be possible to use equation 1 to compute the number of bits required at each selected frequency, and then choose an appropriate tape speed, number of tracks, and duration of the observation to minimize the tape consumption. Unpredictable variations in certain parameters, most notably the correlated flux density and the antenna and receiver performance, would limit how well the minimization could be effected. The savings in tape consumption that could be realized by optinizing each observation must be weighed against the resulting additional operating complexities and associated costs. At present, we have employed a relatively simple scheme in which all of the observations are of the same duration and consume a fixed quantity of tape. This strategy yields a variable SNR which is coupled to the brightness of the source. But the range of variability can be limited by judicious selection of sources, and, as long as the SNR is sufficient, there is little detectable effect of the variations on the reduced observations.

The difference in phase of a single-frequency signal at the two telescopes is ambiguous by multiples of $2 \pi$ radians. The technique used to eliminate this ambiguity is commonly referred to as bandwidth synthesis. This technique consists of measuring the phase difference at a number of frequencies distributed over a sufficiently wide frequency interval for the 
group delay, i.e., the derivative of phase with respect to frequency, to be determined with the desired precision. The group delay uncertainty, $\sigma_{\tau}$, can be calculated with the following equation, also derived by Rogers (1970):

$$
\sigma_{\tau} \simeq \frac{\mathrm{K} \sigma_{\phi}}{2 \pi \mathrm{B}_{w}}
$$

where:

$\mathrm{K}$ is a dimensionless factor that depends on the distribution of the frequencies, and for a nearly optimum distribution is 2 ,

$\sigma_{\phi}$ is the phase uncertainty, and

$B_{W}$ is the spanned bandwidth.

The group delay is itself anbiguous by nultiples of the interval equal to the inverse of the difference between the closest two frequencies observed. For example, if the observed frequencies have a minimum spacing of $25 \mathrm{MHz}$, the ambiguity spacing is $1 /\left(25 \times 10^{6}\right)=40 \times 10^{-9}$ seconds, which is equivalent to approxinately 12 meters in units of length. If it is not carefully chosen to be larger than the largest unmodeled systematic error, the group delay ambiguity spacing can strongly impact the reduction and analysis process.

The options available in selecting the frequencies to be observed are constrained by the performance linitations of the instrumentation. For example, the $\mathrm{X}$-band receivers now in use typically have a usable bandwidth of about $500 \mathrm{MHz}$ or less, which therefore represents the maximum bandwidth that can be selected. The maximum number of frequencies that can be recorded simultaneously is also usually restricted by instrumentation linitations.

The observed delays are contaminated by the differences in delays which the signals suffer in traversing the ionosphere along the separate paths to the two telescopes. Because the ionospheric effect is dispersive, the differences in observed group delays at two sufficiently separated frequency bands, such as X-and S-band, can be used to deduce and remove it. Equation 3 can be used to compute the corrected delay, $\tau_{c}$ :

$$
\tau_{c}=\tau_{x}-\frac{\left(\tau_{x}-\tau_{s}\right) F_{s}^{2}}{\left(F_{s}^{2}-F_{x}^{2}\right)}
$$

where:

$$
\begin{aligned}
& \tau \mathrm{is} \text { the group delay at } \mathrm{X} \text {-band, } \\
& \tau^{\mathrm{x}} \text { is the group delay at } \mathrm{S} \text {-band, } \\
& \mathrm{F}^{\mathrm{S}} \text { is the center frequency at } \mathrm{X} \text {-band, and } \\
& \mathrm{F}_{\mathrm{S}}^{\mathrm{x}} \text { is the center frequency at } \mathrm{S} \text {-band. }
\end{aligned}
$$


Shapiro (1976) has shown that this method of calibration should limit the degradation of ${ }^{\sigma} \tau_{x}$ by the ionosphere to about 7 percent, which corresponds to a few millimeters. If the group delay measurewents at the two frequency bands were accurate enough to allow the phase ambiguity between the bands to be elininated, the correction could be nade even nore accurately, perhaps approaching 1 millimeter. There are, of course, other factors (e.g., the neutral atmosphere) that also affect the observed delays and must be accounted for in reducing the observations. Because these are non-dispersive, they cannot be detected and eliminated by multi-frequency observations, and hence play no role in the selection of observing frequencies.

Taking into account the factors presented above, along with accumulated experience, a set of specifications defining an observation is formulated. To illustrate, we will use the MERIT specifications. The MARK III data acquisition systems deployed during the MERIT campaign did not have full design capabilities, and the number of frequencies that could be observed simultaneously was restricted to 7. Four were assigned to $\mathrm{X}$-band and 3 to $\mathrm{S}$-band. The specific frequencies are listed in table 1. Two tracks were used to record 8 megabits per second at each frequency, and each observation was 110 seconds in duration. After recording the full length of a tape in one direction, the direction was reversed and the renaining 14 tracks were recorded. Of course the tape was stopped between observations while the telescopes were repointed for the next observation. This procedure resulted in a mean time between tape changes of about 2 hours. We emphasize that the constraint of observing a maximum of 7 frequencies caused added complexities in reducing the MERIT data, and we will certainly change the specifications for the POLARIS observations as soon as the instrumentation permits.

Table 1. List of the $4 \mathrm{X}$-Band and $3 \mathrm{~S}$-Band frequencies used during the MERIT Campaign.

\begin{tabular}{|c|c|c|}
\hline \multicolumn{3}{|c|}{ Observing Frequencies (Mhz) } \\
\hline & $X-B a n d$ & S-Band \\
\hline 1 & 8240.99 & 2220.99 \\
\hline 2 & 8340.99 & 2270.99 \\
\hline 3 & 8490.99 & 2295.99 \\
\hline 4 & 8540.99 & \\
\hline
\end{tabular}

Once the format of a single observation has been specified, the next step is to develop an observing schedule which details the sequence of observations to be performed during a session. An algorithm which automatically produces an optimized schedule has not yet been developed. The current procedure involves an operator working with the aid of an interactive scheduling program. The operator responds to program prompts to define an observation, to specify the observatories that will participate in the observing session, and to select the sources to be used. Typically, a set of 10 to 20 sources reasonably distributed in right 
Table 2. List of the 14 sources used for MERIT and POLARIS observations. The coordinates of the sources marked with (*) were sufficiently well known from previous observations that they were held fixed in the solutions presented in this paper.

\begin{tabular}{|c|c|c|c|c|}
\hline & Source & Name & Rt. Asc. & Declination \\
\hline & IAU & Alternate & $\mathrm{h} \quad \mathrm{m} \quad \mathrm{s}$ & d in $\mathrm{s}$ \\
\hline & $0106+013$ & & $\begin{array}{llll}01 & 06 & 04.5181\end{array}$ & $01 \quad 1901.074$ \\
\hline$*$ & $0224+671$ & $4 \mathrm{C67} .05$ & 022441.1339 & $\begin{array}{lll}67 & 07 & 39.338\end{array}$ \\
\hline & $0235+164$ & & $02 \quad 35 \quad 52.6194$ & $\begin{array}{lll}16 & 24 & 03.880\end{array}$ \\
\hline * & $0355+508$ & NRAO 150 & $03 \quad 55 \quad 45.2277$ & $\begin{array}{lll}50 \quad 49 & 20.068\end{array}$ \\
\hline & $0552+398$ & & $05 \quad 52 \quad 01.3732$ & $\begin{array}{lll}39 & 48 & 21.924\end{array}$ \\
\hline * & $0851+202$ & OJ 287 & $0851 \quad 57.2298$ & 201758.596 \\
\hline & $0923+392$ & $4 C 39.25$ & $0923 \quad 55.2943$ & $\begin{array}{lll}39 & 15 & 23.828\end{array}$ \\
\hline * & $1226+023$ & $3 \mathrm{C} 273 \mathrm{~B}$ & $1226 \quad 33.2460$ & $02 \quad 19 \quad 43.470$ \\
\hline * & $1404+286$ & $0 Q 208$ & $1404 \quad 45.6284$ & $28 \quad 41 \quad 29.524$ \\
\hline * & $1641+399$ & $3 C 345$ & $16 \quad 41 \quad 17.6401$ & $\begin{array}{lll}39 & 54 & 10.991\end{array}$ \\
\hline & $1642+690$ & & 164218.1420 & $\begin{array}{lll}69 & 02 & 13.428\end{array}$ \\
\hline * & $2134+004$ & $2134+00$ & 213405.2261 & $00 \quad 28 \quad 25.020$ \\
\hline & $2200+420$ & VR422201 & $2200 \quad 39.3880$ & $\begin{array}{lll}42 & 02 & 08.331\end{array}$ \\
\hline * & $2251+158$ & $3 C 454.3$ & $22 \quad 51 \quad 29.5335$ & $15 \quad 52 \quad 54.184$ \\
\hline
\end{tabular}

ascension and declination is selected, based primarily on brightness, simplicity of structure, and previous usage. Table 2 lists the 14 sources used in the MERIT campaign. The operator specifies a starting time, and the program displays the subset of sources above the network horizon at that time. For continental scale networks, there will usually be 3 to 6 sources visible from all observatories at any given time. The operator need only select the source to be observed, and the program schedules the observation. In scheduling the observations, the program automatically takes into account instrumental constraints, such as the time required for each telescope to be re-pointed after the previous observation, and allocates specific segments of tape (start and stop footages) to each observation. The progran does an excellent job of relieving the operator of the tedious computational and accounting aspects of making the schedule, freeing him to concentrate on the sequence of the observations.

The general strategy in selecting the sequence of observations is to construct a series of observations of each source well distributed over the full range of hour angle that can be observed. It is also desirable to interleave the observations on the different sources in a manner that avoids excessive amounts of time spent slewing the telescopes. Figure 2 is a sample plot showing the sequence of observations as a function of time and declination, which is used to aid the operator in formulating the observing sequence. For networks having a large geometrical extent, it may be desirable to schedule observations on sources which are visible only to a sub-set of the full network. For example, the MERIT network extended over nearly 30 degrees in latitude (HRAS to Onsala) and 130 degrees in longitude (OVRO to Onsala). This extent seriously restricted the range of hour angle over which all the observatories could view certain sources. To supplement the coverage, subnets were formed in which Haystack, OVRO, and 
Table 3. Sample portion of observing schedule used during the MERIT campaign.

\begin{tabular}{|c|c|c|c|c|c|}
\hline \multicolumn{4}{|c|}{ Date } & \multirow{2}{*}{ Source } & \multirow{2}{*}{$\begin{array}{l}\text { Stations } \\
\text { Participating }\end{array}$} \\
\hline $\mathrm{y}$ & $\mathrm{m} d$ & $\mathrm{~h}$ & $\mathrm{~m}$ & & \\
\hline 80 & 927 & 23 & 40 & $1642+690$ & HA HR OV ON EF \\
\hline 80 & 927 & 23 & 49 & $3 C 345$ & HA HR OV ON EF \\
\hline 80 & 927 & 23 & 58 & VR422201 & HA HR OV ON EF \\
\hline 80 & 928 & 0 & 5 & $0106+013$ & HA ON EF \\
\hline 80 & 928 & 0 & 16 & NKA0 150 & $\mathrm{HA}$ ON EF \\
\hline 80 & 928 & 0 & 28 & $0235+164$ & HA ON EF \\
\hline 80 & 928 & 0 & 38 & VR422201 & HA HR OV ON EF \\
\hline 80 & 928 & 0 & 48 & $1642+690$ & HA HR OV ON EF \\
\hline 80 & 928 & 0 & 58 & $3 c 345$ & HA HR OV ON \\
\hline 80 & 928 & 1 & 5 & OQ208 & HA HR OV \\
\hline 80 & 928 & 1 & 22 & VR422201 & HA HR OV ON EF \\
\hline 80 & 928 & 1 & 28 & $3 C 454.3$ & $\mathrm{HA} \mathrm{HR}$ OV ON EF \\
\hline 80 & 928 & 1 & 35 & $2134+00$ & HA HR OV ON EF \\
\hline 80 & 928 & 1 & 46 & $0552+398$ & HA ON EF \\
\hline 80 & 928 & 1 & 55 & NRAO 150 & $\mathrm{HA}$ ON EF \\
\hline 80 & 928 & 2 & 2 & $4 C 67.05$ & HA OV ON EF \\
\hline 80 & 928 & 2 & 10 & $0235+164$ & $\mathrm{HA}$ ON EF \\
\hline 80 & 928 & 2 & 16 & $0106+013$ & HA ON EF \\
\hline 80 & 928 & 2 & 29 & $1642+690$ & HA HR OV ON EF \\
\hline 80 & 928 & 2 & 41 & VR422201 & $\mathrm{HA} \mathrm{HR}$ OV ON EF \\
\hline
\end{tabular}

HRAS made extreme westerly observations, while Haystack and the European observatories made extreme easterly observations. Table 3 contains a sample of the schedule used in the MERIT campaign.

\section{DATA ACQUISITION}

After the schedule has been formulated, the schedules and station instructions are placed on floppy disks to be used by the computers at the observatories to operate the data acquisition systems. These floppy disks are usually mailed to the observatories in advance of the observing session. However, should țey fail to arrive on time, or if last minute changes are required, it is also possible to transfer the necessary infornation via telecommunications. The data acquisition systern is so automated that the operator has few duties beyond changing the tape when alerted by the computer. As long as there are no nalfunctions, an inexperienced person can operate the data acquisition system with only a few hours training.

In addition to the radionetric data collected at the observatories, ancillary data (such as ambient temperature, baronetric pressure, relative humidity, cable calibration data, water vapor radiometer data, and - operator's comments), which constitute the station 10 , a re recorded on a floppy disk. The data tapes and floppy disks are then sent, usually by mail or by air freight, to the correlator facility at Haystack Observatory. 
CORRELATOR OPERATIONS

Only one MARK III correlator presently exists. It was built and is operated by the North East Radio Observatory Corporation (NEROC) at Haystack Observatory, Massachusetts. At present, the correlator has only single baseline capability, but it will eventually be completed to the original design specifications and be able to process three baselines sinultaneously. A pair of tapes from a baseline can be processed through the correlator in roughly the same time interval as the observing session, i.e., one day's observations can be processed in one day. For networks, the number of baselines increases quickly as the number of observatories increases, e.g., 3 observatories yield 3 baselines while 5 observatories yield 10 baselines. The correlator (particularly a single baseline mode1) can easily become the limiting constraint on the number of measurements that can be made.

The delays and delay rates obtained by the correlator are recorded on standard computer magnetic tape (9-track, $800 \mathrm{bpi}$ ) and forwarded to the customer. Archive tapes are also written and placed in storage. The raw data tapes (instrumentation tapes) are usually stored until the customer has processed the observations and notifies the correlator facility that the tapes are cleared for re-use. The tapes are then placed back into inventory and eventually are distributed to observatories for re-use. The mean life of an instrumentation tape is not yet well determined, and a number of different manufacturers' tapes (e.g., Fuji, Sony, Ampex) are being tested.

FINAL REDUCTION AND ANALYSIS OF THE OBSERVATIONS

The observed delays and delay rates received from the correlator have corrections applied to them to account for instrumental and atmospheric effects, and are then combined in a least squares solution to estimate the geodetic parameters. All of the processing is performed with a mini-computer system specifically configured for a high degree of interaction with the operator, including extensive use of interactive computer graphics. The illustrations used in the following discussion were selected from graphic displays routinely produced during the data reduction and analysis process.

Figures 3 and 4 show examples of two calibration corrections applied to the MERIT data. The "cable calibration" accounts for fluctuations in the transit time of the clock signal through the cable connecting the system clock, located in the observatory control center, to the first local oscillator, located in the receiver box on the telescope. The fluctuations, of the order of a few tenths of a nanosecond, are primarily caused by temperature changes in the cable. Figure 3 is a plot of the cable calibration from HRAS for the first week of the MERIT campaign. Note that the fluctuations are greater at the end of the week than at the beginning. This difference resulted from local weather conditions; there was a severe storm during the early part of the week, which limited the 


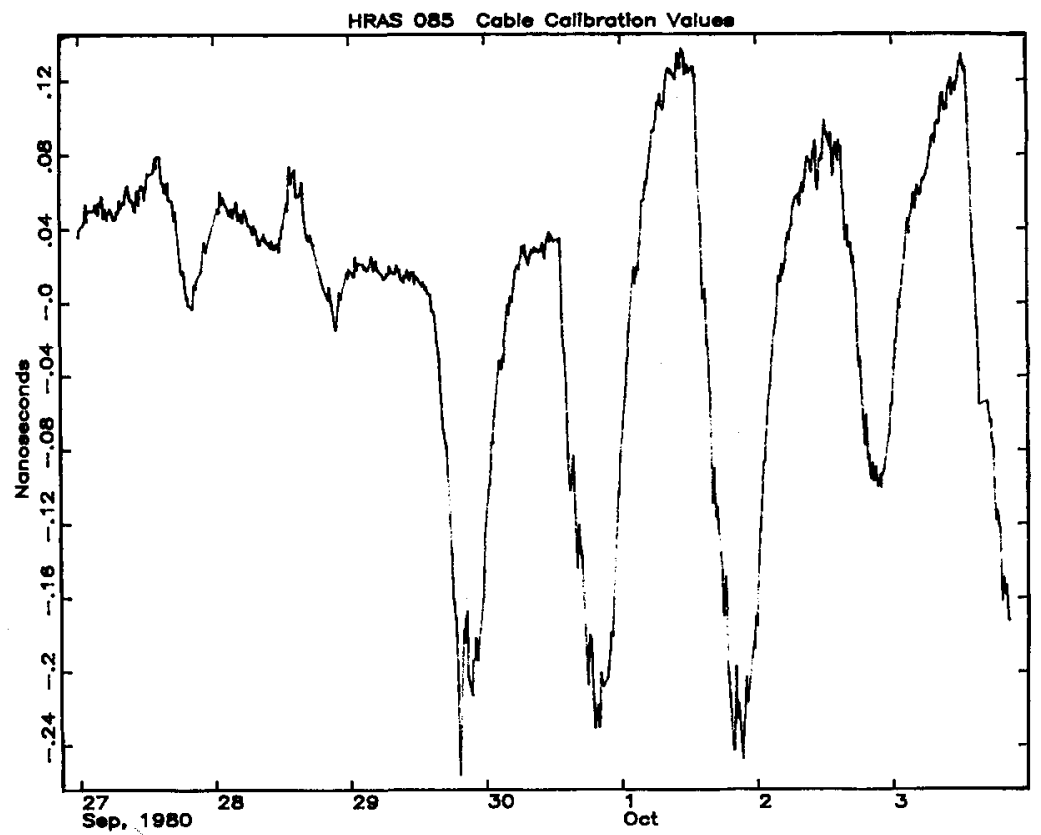

Figure 3. HRAS cable calibration values for the first week of project MERIT.

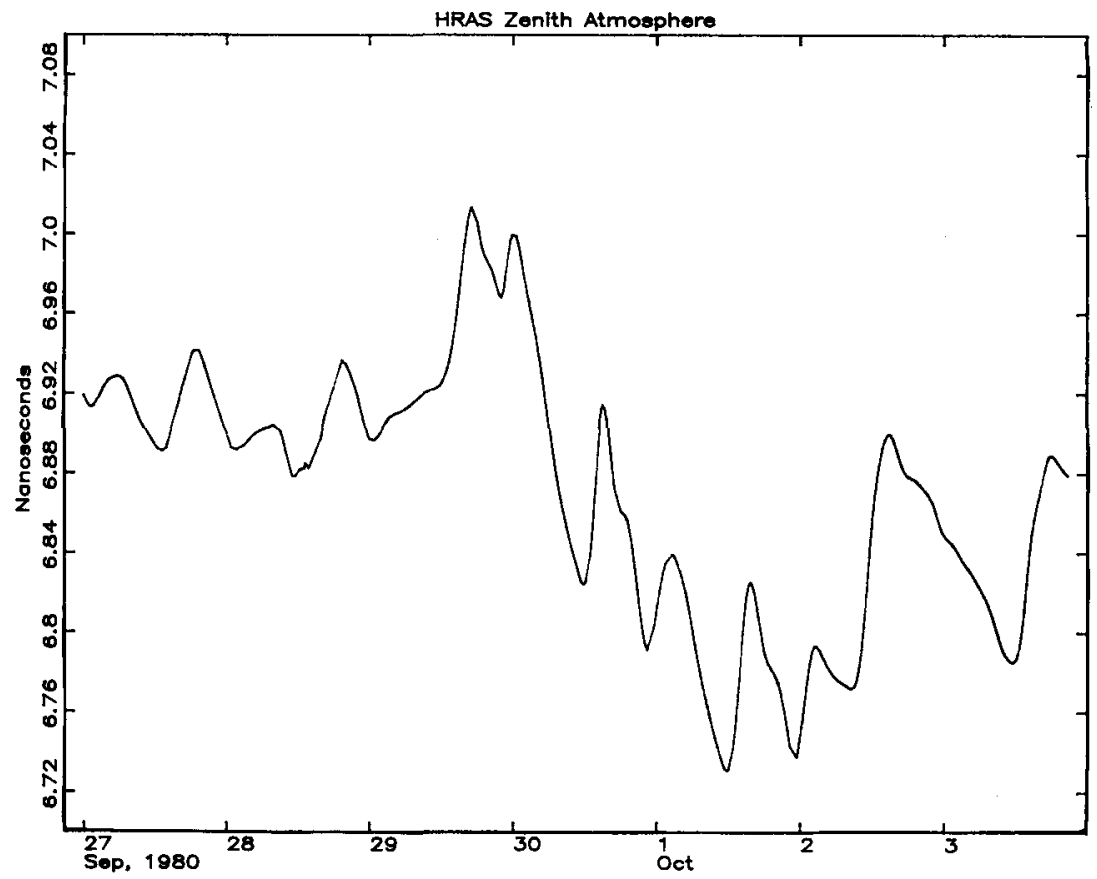

Figure 4. HRAS zenith atmosphere correction. 
ambient temperature fluctuations as well as the exposure of the cable to direct sunlight. All of the stations in the MERIT campaign except Haystack recorded cable calibration data during all of the observing sessions. The lack of such data at Haystack is probably unimportant since the cable at Haystack is under a radome and is not exposed to large ambient temperature fluctuations.

No attempt is made to determine the absolute magnitude of the instrumental delays. For most geodetic applications, constant instrumental delays are indistinguishable from clock offset errors, and have no effect on the recovery of geodetic parameters. Absolute calibration of the instrumental delays is only important for high accuracy time transfer applications (see, e.g., Clark et al., 1979).

The observed delays and delay rates must also be corrected for the differences in the electrical path lengths traveled by the signals through the neutral atmosphere enroute to the telescopes. The absolute delay introduced by the neutral atmosphere is about 6 to 8 nanoseconds at the zenith, depending upon the elevation of the observatory and local weather conditions, and increases approximately as the secant of the zenith angle. Standard equations can be used to estimate the zenith delay with uncertainties of a few decimeters from ambient temperature, barometric pressure, and relative humidity. The remaining uncertainty is dominated by the variable water vapor content of the atmosphere. Theoretical studies and limited experimental testing suggest that water vapor radiometers (WVRs) may be capable of determining that component to a centimeter or better. The MARK III VLBI data acquisition system is designed to include WVRs, but these instruments are not yet in routine operation at most observatories. Only the Onsala Observatory routinely collected WVR data during the MERIT campaign. Some observations were also made at oVRO during the second week of MERIT. No WVR information was used in obtaining the results presented in this paper. In the absence of water vapor radiometry data, an atmospheric model was employed.

Figure 4 shows the atmospheric zenith thickness at HRAS calculated from measurements of the surface pressure, temperature, and humidity, using a model developed by J. Marini at NASA Goddard Space Flight Center (Marini, 1974). All of the stations participating in the MERIT campaign except Onsala and Efflesberg had digital read-out meteorological sensors, whose output was automatically sampled by the Mark III field system computer. At Onsala and Efflesberg the meteorological data were entered into the logs by the operators.

After the cable calibration and neutral atmospheric corrections have been made, the next stage in the processing entails the elimination of the group delay ambiguites, and editing the observations to remove "outlier" data points. Both problems are dealt with initially by examining the residuals of the data to theoretical calculations based on good a priori values for station coordinates, source coordinates, and other parameters affecting the data. A priori values for the clock rate errors can be determined from the delay rate data. A typical set of X-band delay residuals is shown in figure 5. The data cluster into sets which are separated by the group delay ambiguity ( 20 nanoseconds, in this case). Since the errors in the theoretical values are very much smaller than the spacing of the group delay ambiguities, it is a simple matter to determine 


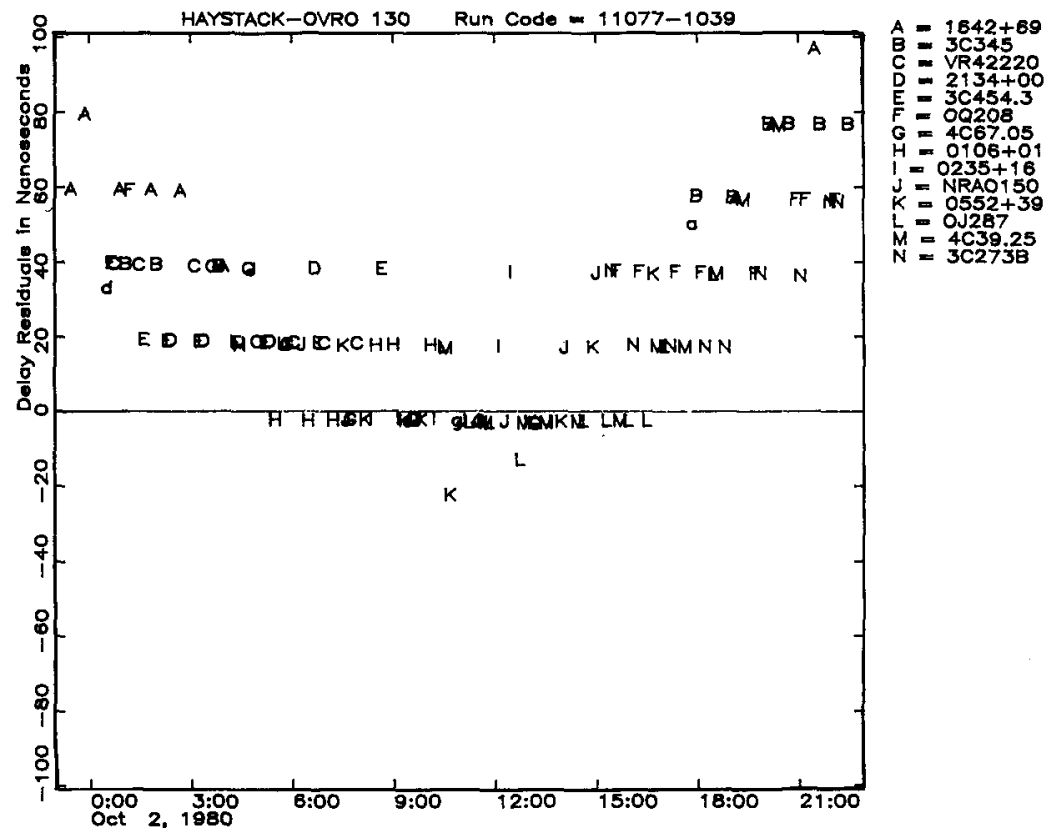

Figure 5. $X$-Band delay residuals froin the Haystack-OVRO baseline showing the effects of unresolved group delay ambiguities.

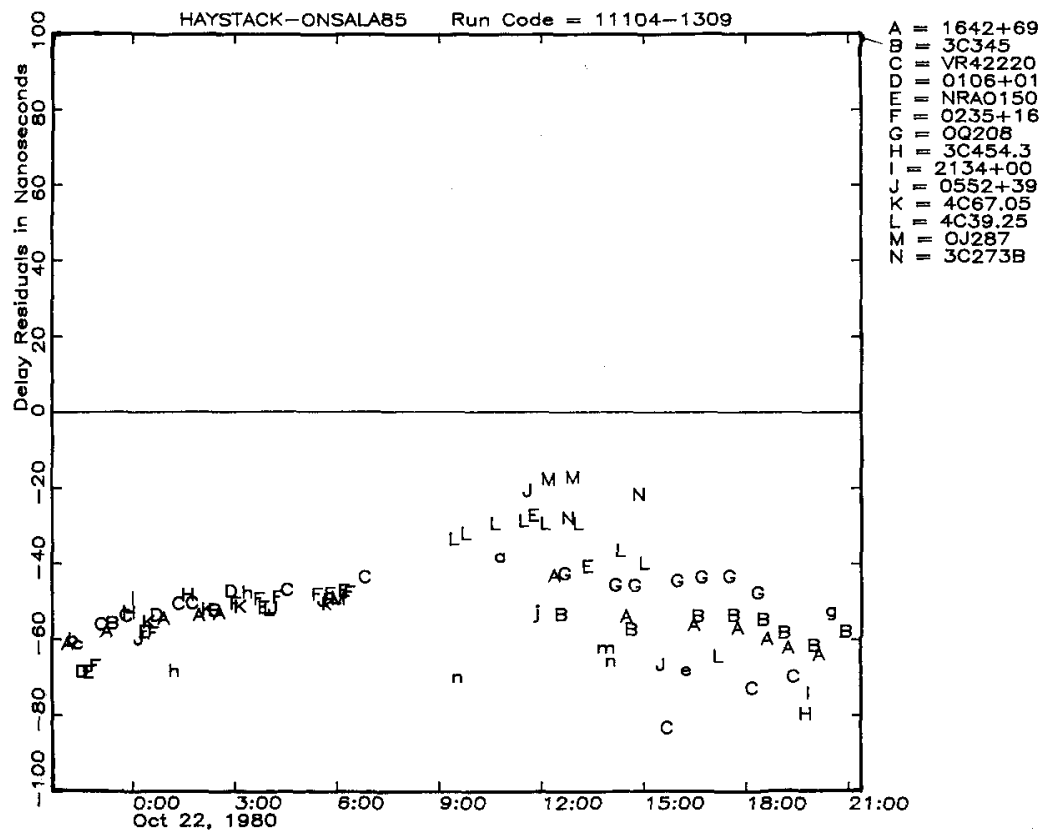

Figure 6. S-Band delay residuals from the Haystack-Onsala baseline showing the effects of ionospheric delays. 
the discrete ambiguity offsets and make the appropriate corrections. The resolution of the S-band ambiguities was made difficult by the fact that we did not have an ionospheric model for use. in calculating the theoretical values, and the ambiguity spacing was of the same order as the ionospheric effects. For the MERIT data, an S-band group delay spacing of 40 nanoseconds had been selected. Figure 6 shows the residuals to a set of S-band data which has no ionospheric correction. The data exhibit a scatter which is small compared to the group delay ambiguity during the night ( 21 h to $9 \mathrm{~h}$ UT), but which becomes comparable to the group delay spacing during the daytime. The ambiguities in these data were successfully resolved only by comparison with the corresponding X-band data and by careful examination of the ionospheric corrections implied by the S-X observation differences.

Figure 7 shows the ionospheric corrections for the Haystack-OVRO $\mathrm{X}$-band delays as determined from the $\mathrm{S}$-band minus $\mathrm{X}$-band differences. The different lines represent sequences of observations on different sources. The effect is seen to vary from about 0 to 3 nanoseconds. Figure 8 shows the corresponding delays for the Haystack-Onsala baseline. Here the effects are slightly larger, ranging from 0 to 4 nanoseconds. Some of the variations in figures 7 and 8 are caused by changes in the observation elevation angles. Nevertheless, it is possible to see the general structure of the day-night variations in the ionosphere in these figures. Also clearly shown in figure 8 is the period during which the $S$-band antenna at Onsala had to cease operations as a result of high winds. Noting the apparent repeatability of the ionospheric correction from day to day, we made a crude attempt to bridge this gap by applying the correction trom the previous day to the data within the gap. Some of the effects of making this correction are discussed under Results. It is clear from figures 7 and 8 that the ionosphere has an effect on the $X$-band observations of one meter or more. If this effect were not removed, some portion of it could be expected to be absorbed into the clock parameter and the atmospheric zenith height parameter estimates. Some portion of it would probably enter the baseline parameter estimates as well. During the MERIT campaign between 600 and 900 observations were scheduled for each day, depending on how many stations were observing. An observation was retained in our solutions only if the signal was reliably detected by the correlator and the residuals of both the $\mathrm{S}-$ and $\mathrm{X}$-band observations were reasonable. In general, 75 to 85 percent of the observations were retained. We are still investigating the causes of the unexpectedly low yield of acceptable observations. The dominant cause seems to have been the lack of adequate sensitivity at S-band on some baselines. Three of the daily periods show a loss of nearly 50 percent of the scheduled observations. In two of these periods the Onsala s-band antenna was forced to suspend operations as a result of high winds; in the third, errors in the pointing software at Haystack caused that antenna to miss all of the observations west of the meridian. (Notice that the loss of a single antenna from a network of $N$ antennas causes a loss of $200 / \mathrm{N}$ percent of the baselines. For small values of $\mathrm{N}$, this percentage is quite large.) In addition, the data from Chilbolton exhibited some instrumental irregularities, including clock and cable calibrator irregularities, which are still being investigated. These problems have delayed the processing 


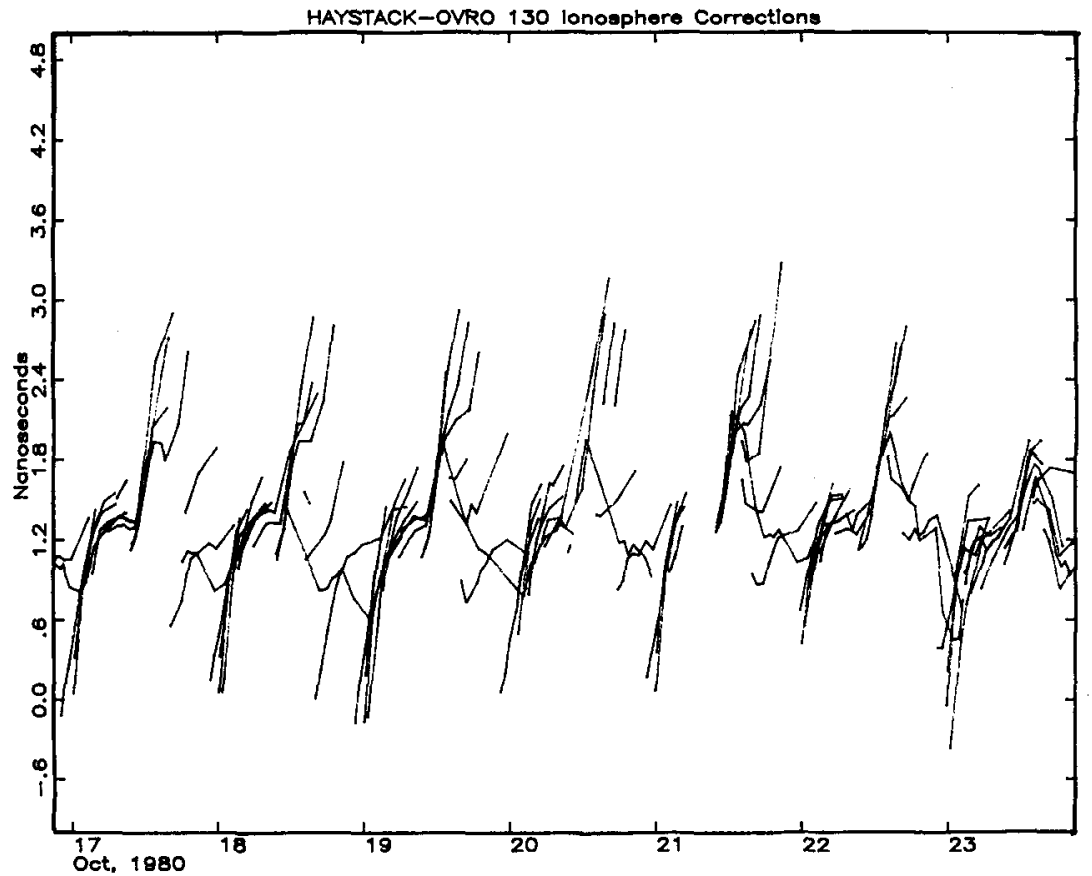

Figure 7. Ionosphere corrections for Haystack-OVRO baseline.

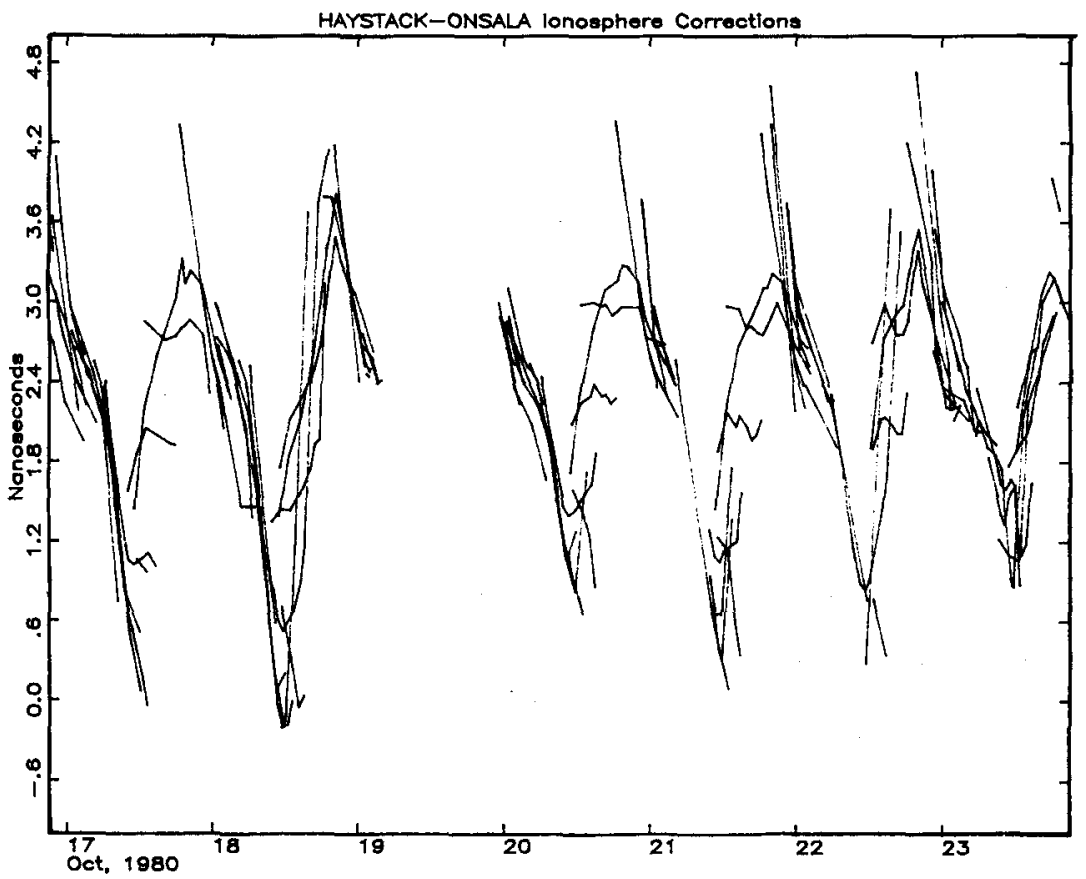

Figure 8. Ionosphere corrections for Haystack-Onsala baseline. 
Table 4. List of the $X$ and $Y$ components of the pole positions in milliseconds of arc and UT1-UTC values in seconds of time determined from the MERIT and POLARIS VLBI observations.

\begin{tabular}{|c|c|c|c|c|c|c|c|c|}
\hline & $\begin{array}{r}\text { te } \\
\mathrm{m}\end{array}$ & $\mathrm{d}$ & $\mathrm{x}$ & $\sigma_{\mathbf{x}}$ & $\mathrm{Y}$ & $\sigma_{y}$ & UT1-UTC & $\sigma_{\mathrm{UT} 1}$ \\
\hline 80 & 9 & 27 & -16.4 & 1.4 & 340.7 & 1.5 & .04098 & .00006 \\
\hline 80 & 9 & 28 & -13.3 & 1.6 & 341.1 & 1.6 & .03797 & .00008 \\
\hline 80 & 9 & 29 & -13.0 & 1.3 & 337.7 & 1.4 & .03522 & .00007 \\
\hline 80 & 9 & 30 & -12.1 & 1.3 & 337.4 & 1.6 & .03264 & .00007 \\
\hline 80 & 10 & 1 & -14.8 & 1.3 & 342.0 & 1.6 & .03042 & .00007 \\
\hline 80 & 10 & 2 & -16.2 & 1.4 & 337.9 & 2.8 & .02825 & .00009 \\
\hline 80 & 10 & 3 & -13.3 & 1.3 & 339.6 & 1.6 & .02596 & .00007 \\
\hline 80 & 10 & 17 & -4.0 & $-\cdots$ & 351.0 & -- & -.00685 & -- \\
\hline 80 & 10 & 18 & -3.0 & 1.2 & 353.8 & 1.4 & -.00933 & .00006 \\
\hline 80 & 10 & 19 & -4.1 & 1.0 & 353.9 & 1.8 & -.01182 & .00007 \\
\hline 80 & 10 & 20 & $-4 \cdot 6$ & 1.3 & 357.2 & 1.3 & -.01443 & .00006 \\
\hline 80 & 10 & 21 & -4.7 & 1.1 & 355.3 & 1.3 & -.01725 & .00005 \\
\hline 80 & 10 & 22 & -7.3 & 1.2 & 354.8 & 1.5 & -.02017 & .00006 \\
\hline 80 & 10 & 23 & -4.6 & 1.1 & 355.3 & 1.3 & -.02338 & .00005 \\
\hline 80 & 12 & 2 & 42.2 & 2.0 & 365.1 & 1.7 & -.12443 & .00009 \\
\hline 80 & 12 & 20 & 65.0 & 1.3 & 359.0 & 1.1 & -.16822 & .00007 \\
\hline
\end{tabular}

of the Chilbolton data. None of the Chilbolton observations were used in the analyses reported here.

\section{RESULTS}

The VLBI data were used to determine variations in both the $X$ and $Y$ components of the position of the pole, and UTl-UTC. The Bureau International de 1 'Heure (BIH) Circular D values for October 17, 1980 were adopted to define the origin, and $B I H$ values were also used as a priori values in the VLBI solutions. Table 4 lists the final pole positions, UT1-UTC values, and the formal standard errors for the VLBI solutions. Note that the formal errors of the $X$ and $Y$ components range from $1.1 \mathrm{msec}$ of arc to as large as $2.8 \mathrm{msec}$ of arc (for the $Y$ component of the pole during the time in which the the Onsala S-band antenna was inoperable because of high winds) and were typically about $1.5 \mathrm{msec}$ of arc. The formal errors of the UTI-UTC determinations were less variable, clustering about 0.07 milliseconds. Figure 9 is a plot of the VLBI [crosses], BIH [dotted line] and smoothed Doppler Polar Motion Service (DPMS) [dashed linel pole positions. The DPMS data were smoothed by convolution with a Gaussian function whose full width at half maximum was about 17 days, and shifted to match the VLBI and BIH value on October 17. There is reason to believe that the data from the DPMS are of poorer quality than the comparable data obtained a few years ago. The scatter in the raw data increased in early 1979 when satellite $\# 77$ ceased to be usable. The effect of the loss of this satellite is seen in the increase (by about a 


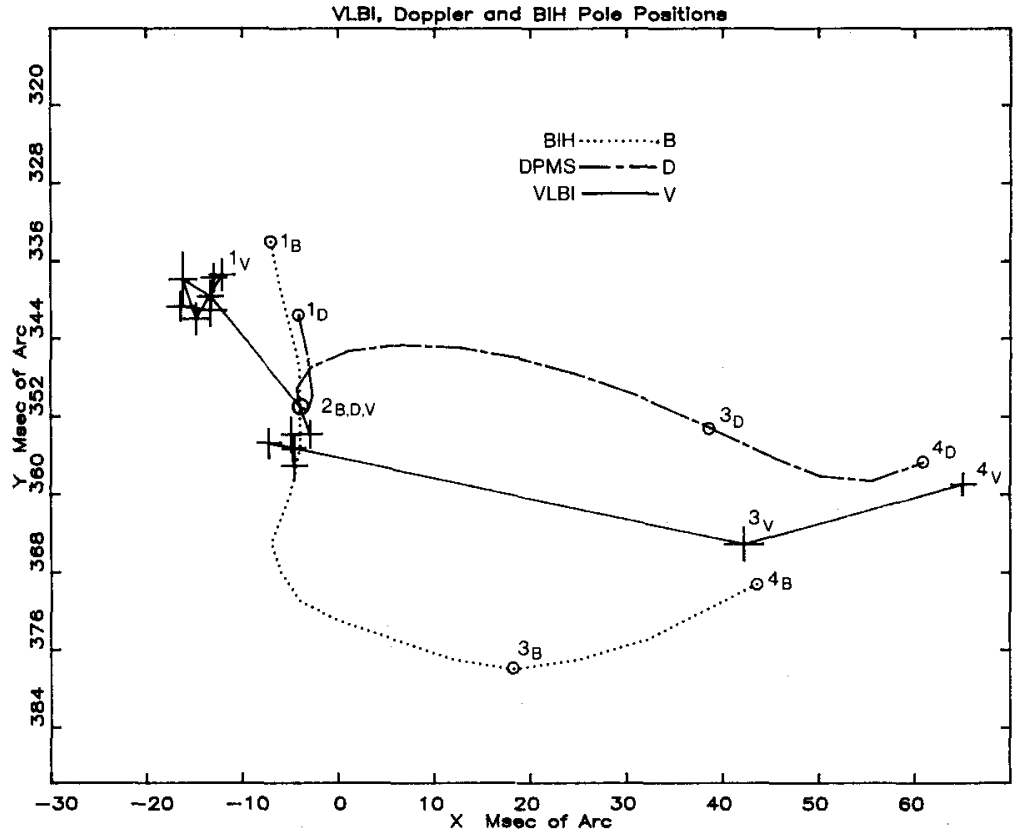

Figure 9. Pole positions from VLBI (crosses), smoothed DPMS (dashed line), and the BIH (dotted line). The numbered points indicate common dates.

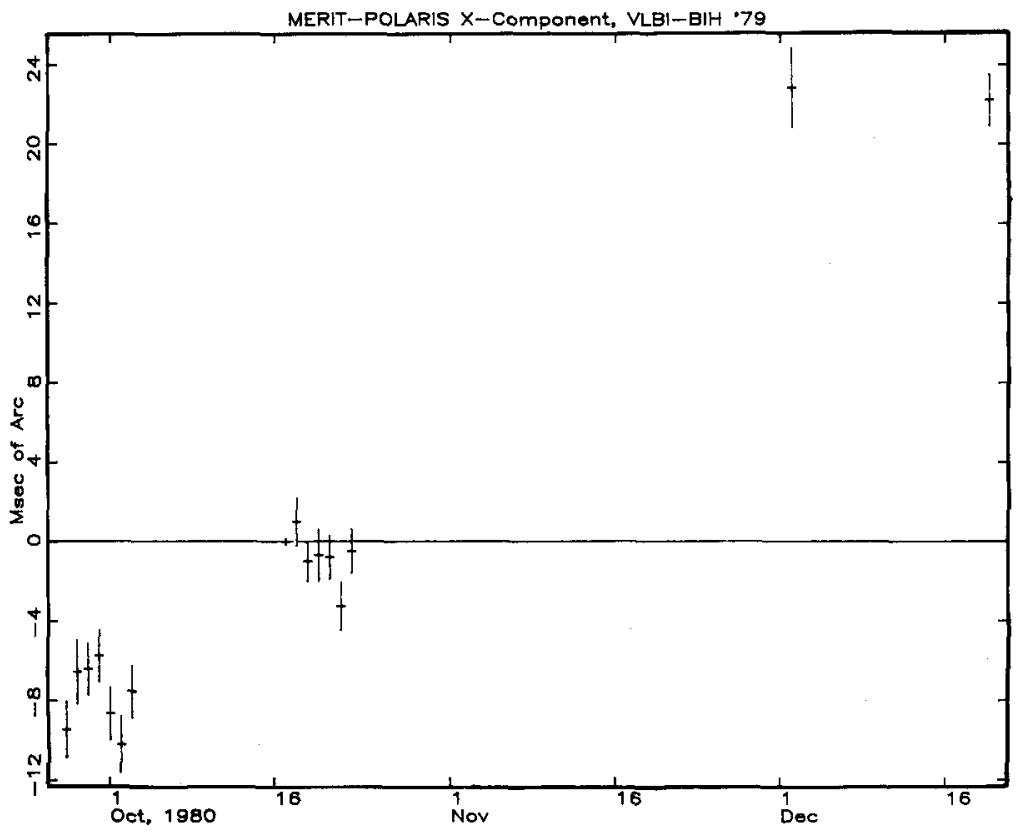

Figure 10. Pole X-component, VLBI determinations minus BIH. 
factor of three) in the pole position errors quoted by the DPMS. In addition, the increased ionospheric activity at solar maxinum is expected to have a deleterious effect on the L-band satellite signals. In spite of these problems, the differences between the VLBI and the DPMS values are only at the level of 10 to $15 \mathrm{msec}$ of arc.

The differences between the VLBI and BIH X values are plotted in figure 10. The VLBI values drift away from the BIH values by about $35 \mathrm{msec}$ over this period. Figure 11 is a comparable plot for the $\mathrm{Y}$ component, and displays a drift of about $20 \mathrm{msec}$. These drifts are consistent with the expected errors in the BIH values (cf. Robertson et al., 1979b). We have included in figure 11 the results of two different VLBI solutions for October 19 , a day on which very little S-band data were available for Onsala. The value plotted as a dashed line is the result obtained using the previous day's ionospheric corrections for Onsala, while the value plotted as a solid line is the result obtained by deleting the Onsala data for which there is no s-band from the solution. The difference, approximately $25 \mathrm{~cm}$, is about four times the combined formal errors of the two estimates. It is possible that a better way can be found to bridge this gap in the data, perhaps by averaging the remaining ionospheric corrections over a several day interval. These possibilities remain to be explored.

Because of the manner in which the BIH processes its data, their results are quite smooth over a period of a week. If we further assume that the behavior of the Earth itself is smooth over the same time period, then the residuals to a smooth curve fit to the differences shown in figures 10 and 11 can be used to obtain a measure of the day-to-day noise in the VLBI estimates. We therefore fitted a parabola to each week of values from the MERIT data and found that the residuals to those parabolas had a root-mean-square (RMS) value of $1.1 \mathrm{msec}$ of arc in $X$ and $1.2 \mathrm{msec}$ of arc in $Y$. These RMS values are consistent with the formal errors in the VLBI data, and indicate that the small scale structure seen in figures 10 and 11 is at about the level expected from the noise level of the VLBI estimates.

Figure 12 displays the difference between the VLBI and the BIH determinations of UTl. The BIH values used to form these differences included the Circular $D$ values and the fortnightly and monthly terms calculated by Woolard (1959). The VLBI determinations exhibit very smooth deviations from the BIH values with an amplitude of about $2 \mathrm{msec}$ of time. These deviations are consistent with the error level in the BIH determinations as reported in Robertson et al. (1979b). Again a check on the short term error level in the VLBI results can be obtained by examining residuals to smooth curves fitted to the points in figure 12. The RMS value of the residuals to two parabolas fit to the two one-week MERIT data sets is $0.05 \mathrm{msec}$ of time, again quite consistent with the formal errors in the VLBI values. The scatter of the polar motion and UT1 estimates about the fitted smooth curves suggests the absence of systematic error effects in the VLBI data with periods substantially less than a week, since the short period scatter is consistent with the magnitudes of the calculated formal errors.

A better check on the total errors in the polar motion and UT1 estimates can be made by performing simultaneous estimates of these 


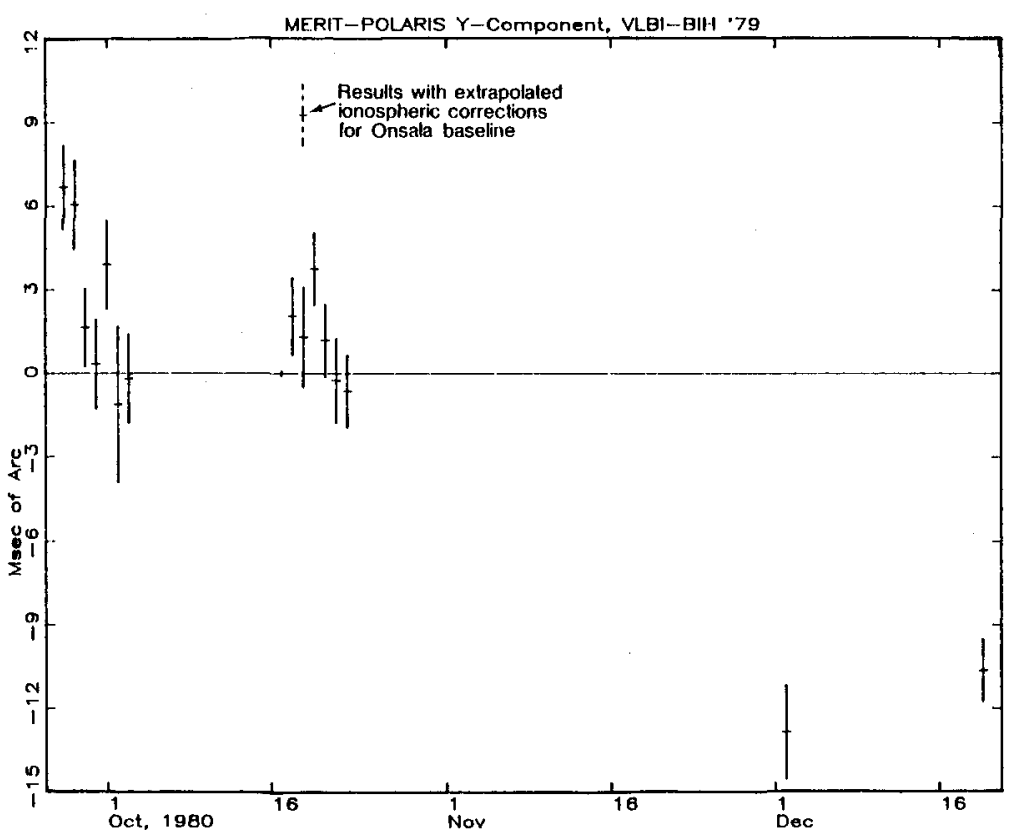

Figure 11. Pole Y-component, VLBI determinations minus BIH.

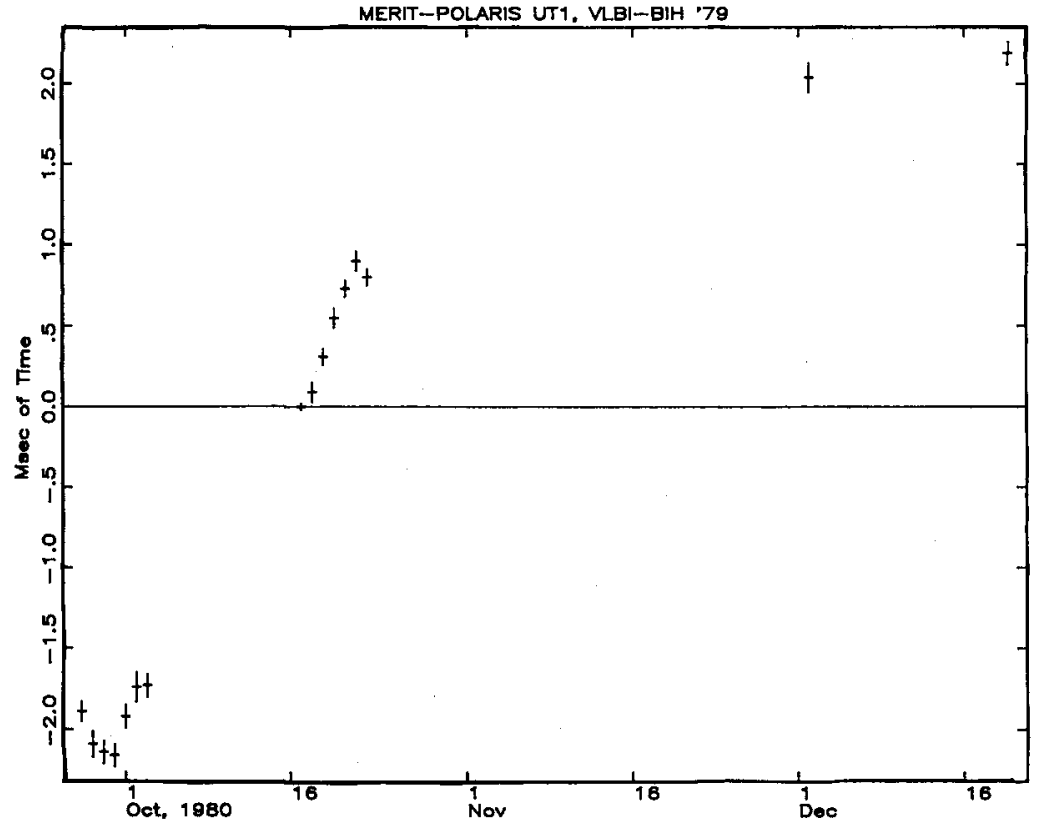

Figure 12. UT1, VLBI determinations minus BIH. The BIH values include the short period Woolard correction terms (see text). 
Table 5. List of the differences between the VLBI solutions for pole position and UTl for two simultaneous triangles (see text), the rootsum-squared (RSS) formal errors, and the ratios (R) of the differences to the RSS values.

\begin{tabular}{|c|c|c|c|c|c|c|c|c|c|c|}
\hline \multicolumn{2}{|c|}{ Date } & \multicolumn{2}{|l|}{$\bar{x}$} & \multirow[b]{2}{*}{$\mathbf{R}$} & \multicolumn{2}{|c|}{$\mathrm{Y}$} & \multicolumn{4}{|c|}{ UT1-UTC } \\
\hline y & m d & Diff & RSS & & Diff & RSS & $\mathrm{R}$ & Diff & RSS & $\mathrm{R}$ \\
\hline 80 & $\begin{array}{ll}9 & 28 \\
9 & 20\end{array}$ & $8.3 \mathrm{~ms}$ & $4.8 \mathrm{~ms}$ & 1.7 & $3.1 \mathrm{~ms}$ & $4.5 \mathrm{~ms}$ & 0.69 & $.00016 \mathrm{~s}$ & $.00021 \mathrm{~s}$ & .76 \\
\hline 80 & 929 & 3.5 & 3.6 & 0.97 & 4.7 & 3.9 & 1.2 & .00010 & .00018 & .56 \\
\hline
\end{tabular}

parameters using independent baseline triangles. During the first three days of the MERIT campaign, there were two stations operating in Europe, one in the eastern United States, and two in the western United States. It was therefore possible to separate the data into two sets, each encompassing a separate intercontinental triangle of roughly equal geographic extent. The first such triangle involved the stations at Efflesberg, Haystack, and HRAS; the second involved the stations at Onsala, Haystack, and OVRO. The triangles were selected this way in order to match the smaller European antenna (Onsala) with the larger western U.S. antenna (OVRO). BIH values were used together with data from one of the three days to define the origin of the pole position and UTl. The differences in the estimated pole positions for the remaining two days are displayed in table 5, along with the root-sum-squared (RSS) formal errors for the two determinations, and the ratios of the differences to the RSS errors. Because only three days of data were employed, the formal errors from these solutions are somewhat larger than the corresponding errors from the solutions employing all of the MERIT and POLARIS data. It is clear from table 5 that the differences between the pole positions and UTl values from the two triangles are quite comparable to the RSS formal errors. In fact the RMS value of their ratios is only 1.04. Since this sample included only 6 points, it hardly represents statistically conclusive evidence that the real errors in the solutions are comparable to the formal errors. Also the two data sets, even though they contained no common baselines, were probably not free of common systematic errors. Effects of the atmosphere at Haystack, for example, were present in both data sets. Nevertheless, this test is at least consistent with the hypothesis that the VLBI results are free of systematic errors much larger than their formal errors. Further testing along these lines, to provide more complete statistical samples and more completely independent data sets is clearly indicated, and should provide one of the best determinations of the true error level in the VLBI Earth rotation determinations. When the Chilbolton data are completely processed, for example, it should be possible to construct separate intercontinental triangles on 6 additional days.

Another useful check on systematic errors in the VLBI data can be obtained by examining the repeatability of the estimates of parameters which are not expected to vary over the period of the observations, e.g. baseline lengths. While it is clear that on some scale the baseline lengths are not truly time invariant, over short time periods and in the absence of significant seismic disturbances in the vicinity of the 
Table 6. List of the weighted mean baseline length determinations and their respective RMS scatter about the mean for $\mathrm{N}$ independent determinations.

\begin{tabular}{lllll}
\hline \multicolumn{2}{c}{ Baseline } & Length & RMS & N \\
Haystack & -Onsala & $5599714.543 \mathrm{~m}$ & $.022 \mathrm{~m}$ & 16 \\
Haystack & -HRAS & 3135641.034 & .026 & 16 \\
Haystack & -OVRO 130 & 3928881.646 & .017 & 14 \\
Onsala & -HRAS & 7940732.321 & .072 & 16 \\
Onsala & -OVRO 130 & 7914131.103 & .048 & 14 \\
HRAS & -OVRO 130 & 1508195.375 & .017 & 14 \\
\hline
\end{tabular}

stations, this assumption is probably reasonable. Table 6 shows the RMS repeatability of the lengths of the 6 baselines which have have a statistically significant number (N) of independent determinations from the MERIT and POLARIS data sets. Each solution incorporated about 24 hours of data, and included estimation of the baseline coordinates, a clock polynomial model determined from an examination of the post-fit residuals, an atmospheric zenith height at each station, and the coordinates of 6 of the 14 sources observed. The remaining 8 sources had adequate a priori coordinate values. It can be seen from table 6 that the baselines up to about $6000 \mathrm{~km}$ exhibit an RMS scatter of only $2-3 \mathrm{~cm}$, and that the $8000-\mathrm{km}$ baselines show a scatter of $5-7 \mathrm{~cm}$. The increased scatter of the lengths of the longer baselines is probably caused by the decreased mutual visibility of sources seen from such distant points and the concomitant decreased hour angle coverage. These values of RMS scatter are slightly larger than the corresponding formal errors of the individual baseline length estimates, which are typically 1-2 $\mathrm{cm}$ for the shorter baselines and 3-5 cn for the longer baselines. Again, we see that there is no evidence of errors substantially in excess of the errors expected from the formal error estimates. It is, of course, dangerous to extrapolate from the behavior of the errors in baseline lengths to the behavior of errors in baseline orientation; the two types of parameters may be affected by different systematic errors. Nevertheless the examination of baseline length repeatability provides a useful check on the overall quality of the VLBI parameter estimation procedures.

It should be noted that the value for the Haystack-OVRO baseline falls within $2 \mathrm{~cm}$ of the value previously published in Robertson et al. (1979a), which was based on data taken from 1976 to 1978 . This would seem to indicate that to within the noise of the measurements there has been no motion across this trans-continental baseline in about four years. The value for the Haystack-Onsala baseline is about $12 \mathrm{~cm}$ different from the value published in Herring et al. (1981) which is based on data taken in 1977 and 1978. Although this difference is more than four times the formal standard error quoted, it is also within the scatter quoted for separate solutions which were constructed from subsets of the data. Some portion of the difference results fron the absence of ionospheric corrections in the earlier results, and the difference should therefore not be interpreted as evidence of actual motion along this baseline. 
Figure 13 shows the residuals of the individual one-day solutions for the Haystack-Onsala baseline. Notice that the error bars are larger on the two days (Oct 1 and Oct 19) when the Onsala S-band antenna was shut down. Figure 14 shows the corresponding plot for Haystack-oVRO. The outlier on this plot (September 27) probably results from the error in the pointing program at Haystack on that day, which, as noted before, caused Haystack to miss all of the observations west of its meridian. It is not surprising to see that this error had a larger effect on the baselines west of Haystack than on baselines east of Haystack.

Examination of the scatter of the baseline lengths has therefore detected no noise sources substantially in excess of that expected from the formal error calculations. Still, a conservative estimate of the true errors in the VLBI parameter estimates would probably be in the vicinity of two to three times the formal errors.

\section{CONCLUSIONS}

The MERIT and POLARIS observing campaigns have been highly successful not only in meeting the immediate goal of collecting a series of VLBI determinations of polar motion and UTl for intercomparison with those produced by other methods, but perhaps even more important, in clarifying the areas where we should focus our efforts to further refine procedures. We have already made several improvements in the MERIT procedures and have identified others that will be implemented in the POLARIS program as soon as instrumental and software capabilities become available. It may be useful to cite a few specific examples. The observing schedules used during MERIT included a number of observations at altitudes of less than 10 degrees, as viewed from one or more of the observatories. A few were even less than 5 degrees. An excessive number of these low altitude observations displayed large residuals, which were almost certainly caused by inadequate atmospheric refraction corrections. We are now restricting the observations to altitudes greater than 10 degrees. In addition we mentioned earlier that only 7 frequencies could be used in the MERIT observations because of instrumental constraints. Three were assigned to the S-band. The frequency spacings selected resulted in an ambiguity spacing of 40 nanoseconds. Unfortunately, the ionospheric induced delays caused the S-band observations to scatter during daylight hours by 40 to 60 nanoseconds, making it extremely tedious to resolve the ambiguities. We are now using a different set of frequencies which yield an ambiguity spacing of 100 nanoseconds, at the cost of an increase in the group delay uncertainty. By mid-sumer of 1981 , we should have the instrumental capacity to observe as many as 14 frequencies simultaneously, and will therefore be able to set the S-band ambiguity large enough to avoid difficulties in resolving the ambiguities, without sacrificing precision in the group delay observable. One source selected for observation, 3c273, was apparently near enough (directionally) to the Sun during the period of the MERIT campaign that its S-band signals were adversely affected by the solar corona. Many such observations were lost. Care should be taken to avoid the scheduling of observations within 15 degrees of the Sun. Several 


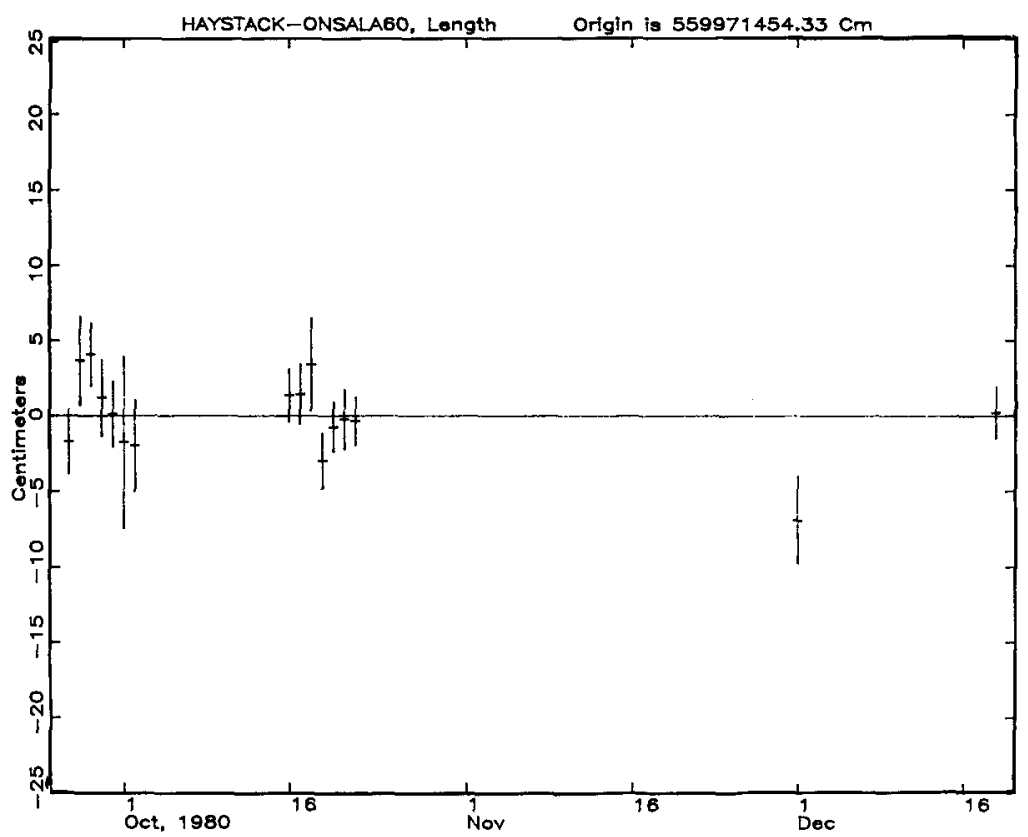

Figure 13. Differences from the mean of the VLBI determinations of the Haystack-Onsala baseline length.

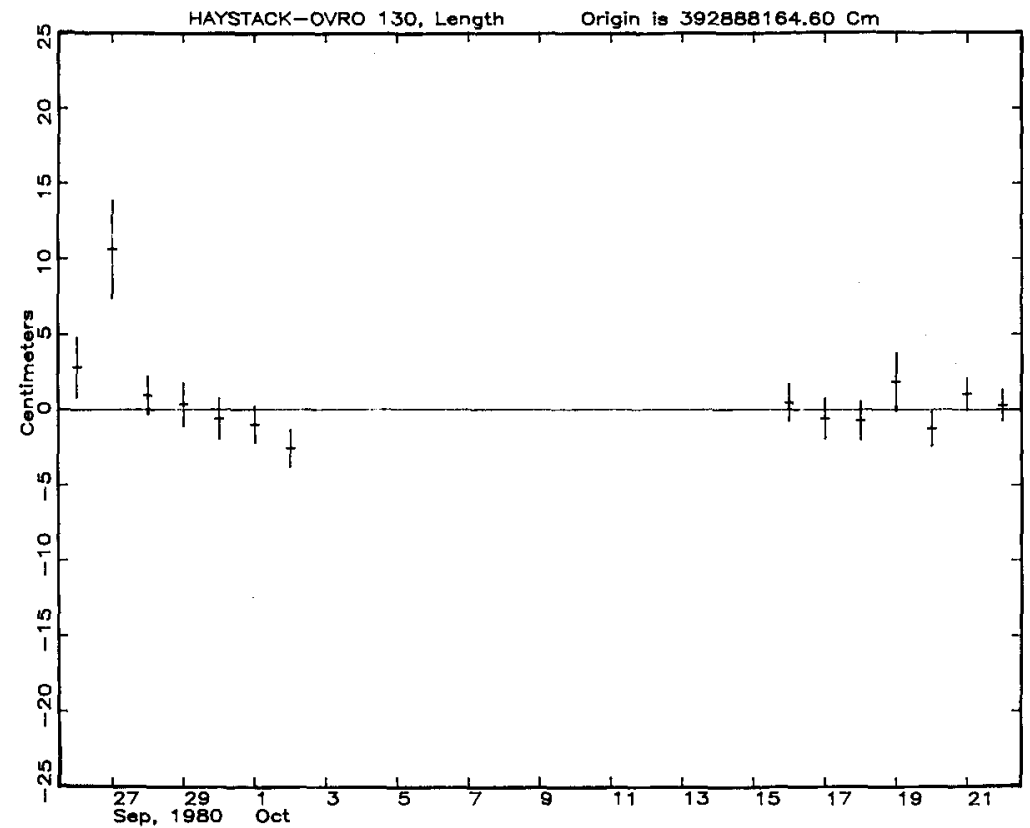

Figure 14. Differences from the mean of the VLBI determinations of the Haystack-OVRO basellne length. 
"bugs," deficiencies, and inconveniences were found in the software. For example, a bug was found in the program that controlled the pointing of the Haystack telescope which caused the telescope to be pointed incorrectly under certain circumstances. About 500 observations were lost during the first few days of the MERIT campaign before this problem was detected and corrected. In spite of these and other problems, the objectives of these observing sessions were fully met. The fact that excellent results were achieved in spite of less than optimal instrumental configurations, observing schedules and operating procedures, speaks well for the robustness of the method.

We expect to continue regular observations at least twice a month for the foreseeable future. Westford Observatory (the second POLARIS station, which began operations in April, 1981) and HRAS will participate in all of these observations, and we intend to include additional stations as of ten as possible. This observation series should provide further valuable intercomparisons of polar motion determinations, as well as providing an opportunity to refine the procedures required to run a continental-scale interferometer on an operational basis.

The prospects for further work using simultaneous baseline triangles to make independent determinations of polar motion and UTl are particularly exciting. Such simultaneous determinations should yield improved estimates of the real error level in the VLBI pole position and UT1 values. It may even be possible to detect effects such as personal biases of the individual experimenters by processing the simultaneous experiments in a "mutually blind" mode; that is, the triangles could be processed independent1y by separate experimenters each of whom is kept unaware of the others' results until the final results are ready for presentation. Ideally the triangles used for such experiments should be totally separated, and even located in separate hemispheres, although this may be impractical in the immediate future. Even the type of experiment reported here, in which both triangles had a single station in common, provide an interesting test of the VLBI results.

In summary, then, the MERIT and POLARIS activities have succeeded in producing very high quality polar motion and UTl information, and have been very useful in uncovering and eliminating many operational problems in the VLBI procedures. These operations have pointed the way to future progress in.this area, and there is good reason to expect to see such progress in the near future.

\section{ACKNOWLEDGMENTS}

The activities reported in this paper could not have been accomplished without the synergistic collaboration of several organizations and many individuals. It is not possible here to detail the contributions of each. We would like to emphasize the critical role of the close cooperation and support provided by the NASA Goddard Space Flight Center, Haystack Observatory, and Massachusetts Institute of Technology VLBI group. Individually, we wish to acknowledge the contributions made by: E. A. Flinn, C. Finley, and T. L. Fischetti, NASA Headquarters; R. J. Coates, T. A. Clark, C. Ma, J. W. Ryan, and W. T. Wildes, NASA 
Goddard Space Flight Center; C. A. Knight, D. B. Shaffer, and N. R. Vandenberg, Phoenix Corporation; B. R. Schupler, Computer Sciences Corporation; J. V. Evans, A. E. E. Rogers, A. R. Whitney, H. F. Hinteregger, J. Webber, and staff members, Northeast Radio Astronomy Corporation, Haystack Observatory; B. E. Corey, C. C. Counselman, T. A. Herring, I. I. Shapiro, Massachusetts Institute of Technology; B. 0. Ronnang, G. Lundquist, and staff members, Onsala Space Observatory; D. H. Eckhardt, Air Force Geophysics Laboratory; H. Seeger and J. Campbell, Bonn University, and staff members, Effelsberg Observatory; J. A. Ball and staff members, Harvard Radio Astronomy Station; R. E. Vogt, A. Readhead, and staff members, Owens Valley Radio Observatory; P. J. Richards, Rutherford and Appleton Laboratories, and staff members, Chilbolton Observatory; M. D. Abell, J. R. Mackay and J. D. Williams, National Geodetic Survey.

\section{REFERENCES}

Carter, W. E., and W. E. Strange, "The National Geodetic Survey Project POLARIS," Tectonophysics, 52, 39-46, 1979.

Carter, W. E., D. S. Robertson, and M. D. Abe11, "An Improved Polar Motion and Earth Rotation Monitoring Service Using Radio Interferometry," in Time and the Earth's Rotation, D. D. McCarthy and J. D. Pilkington, (eds.), D. Reide1 Co., Dordrecht, Holland, 191-197, 1979.

Carter, W. E., "Project POLARIS: a Status Report," in Radio Interferometry Techniques for Geodesy, NASA Conference Publication 2115, 455-460, 1979.

Clark, T. A., C. C. Counselman, P. G. Ford, L. B. Hanson, H. F. Hinteregger, W. J. Klepczynski, C. A. Knight, D. S. Robertson, A. E. E. Rogers, J. W. Ryan, I. I. Shapiro, and A. R. Whitney, "Synchronization of Clocks by Very-Long-Baseline Interferometry," IEEE Transactions on Instrumentation and Measurement, 1979.

Herring, T. A., B. E. Corey, C. C. Counselman III, I. I. Shapiro, B. O. Ronnang, O. E. H. Rydbeck, T. A. Clark, R. J. Coates, C. Ma, J. W. Ryan, N. R. Vandenberg, H. F. Hinteregger, C. A. Knight, A. E. E. Rogers, A. R. Whitney, D. S. Robertson, and B. R. Shupler, "Geodesy by Radio Interferometry: Intercontintental Distance Determinations with Subdecimeter Precision," J. Geophys. Res., 86, 1647-1651, 1981.

Marini, J., private communication, 1974.

Robertson, D. S., W. E. Carter, B. E. Corey, W. D. Cotton, C. C. Counselman, I. I. Shapiro, J. J. Wittels, H. F. Hinteregger, C. A. Knight, A. E. E. Rogers, A. R. Whitney, J. W. Ryan, T. A. Clark, R. J. Coates, C. Ma, and J. M. Moran, "Recent Results of Radio Interferometric Determinations of a Transcontinental Baseline, Polar Motion, and Earth Rotation," in Time and the Earth's Rotation, D. D. McCarthy and J. D. Pilkington, (eds.), D. Reidel Co., Dordrecht, Holland, 217-224, 1979a. 
Robertson, D. S., T. A. Clark, R. J. Coates, C. Ma, J. W. Ryan, B. E. Corey, C. C. Counselman, R. W. King, I. I. Shapiro, H. F. Hinteregger, C. A. Knight, A. E. E. Rogers, A. R. Whitney, J. C. Pigg, B. R. Shupler, "Polar Motion and UTl: Comparison of VLBI, Lunar Laser, Satellite Laser, Satellite Doppler, and Conventional Astrometric Determinations", in Radio Interferometry Techniques for Geodesy, NASA Conference Publication 2115, 33-44, $1979 \mathrm{~b}$.

Rogers, A. E. E., "Very-Long-Baseline Interferometry with Large Effective Bandwidth for Phase-Delay Measurements," Radio Science 5, 1239-1247, 1970.

Shapiro, I. I., "Estimation of Astrometric and Geodetic Parameters," in Methods of Experimental Physics, M. L. Meeks, (ed.), 12, part C, Academic Press, pp 261-266, 1976.

Woolard, E. W., Astronomical Journal, 64, 140-142, 1959.

\section{DISCUSSION}

Mulholzand : Why do alz of your frequencies end in .99?

Reply by Langley : The RF frequencies used in MkIII VLBI observations are usually chosen to be of the form XXXX.99 $\mathrm{MHz}$ in order that the recorded $1 \mathrm{MHz}$ phase calibration signal can be readily extracted at playback.

Klepczynski: How many unknowns do you solve in your solutions for $P M$ and UT and how stable are they with respect to PM and UT ?

Carter: The number of unknowns varies from solution to solution depending on the number of observatories participating, clock models, etc... For the MERIT solution (mostly 4 observatories) the number was about 60 or so. Generally, between solutions with different but "reasonable" sets of parameters in solution, the PM components vary by only a few milliarcseconds (i.e. about $10 \mathrm{~cm})$.

Langley : I understand that your results for the MERIT period were obtained from the same data set as that currently being analyzed by the VLBI group at the Goddard space Flight Center. Could you comment on the differences between their technique and yours and state the magnitude of the resulting differences in the values for UTI and pole position?

Carter : The data set and software used by NGS and GSFC was essentially identical. The primary difference in the solutions is in the number of source coordinates held fixed. NGS held 8 of the source positions fixed, solving for those of the remaining 6 . GSFC solved for fewer source coordinates. I believe they held 12 of the 14 source fixed. The difference in polar motion values were at the 1 to $2 \mathrm{milliarcsecond} \mathrm{level,} \mathrm{and} \mathrm{the} \mathrm{UT} 1$ about 0.1 millisecond of time. 